\title{
ON BASIC AND BASS QUATERNION ORDERS
}

\author{
SARA CHARI, DANIEL SMERTNIG, AND JOHN VOIGHT
}

(Communicated by Benjamin Brubaker)

\begin{abstract}
A quaternion order $\mathcal{O}$ over a Dedekind domain $R$ is Bass if every $R$-superorder is Gorenstein, and $\mathcal{O}$ is basic if it contains an integrally closed quadratic $R$-order. In this article, we show that these conditions are equivalent in local and global settings: a quaternion order is Bass if and only if it is basic. In particular, we show that the property of being basic is a local property of a quaternion order.
\end{abstract}

\section{INTRODUCTION}

Orders in quaternion algebras over number fields arise naturally in many contexts in algebra, number theory, and geometry - for example, in the study of modular forms and automorphic representations and as endomorphism rings of abelian varieties. In the veritable zoo of quaternion orders, authors have distinguished those orders having favorable properties, and as a consequence there has been a certain proliferation of terminology. In this article, we show that two important classes of orders coincide, tying up a few threads in the literature.

Setup. Let $R$ be a Dedekind domain and let $F$ be its field of fractions. Let $B$ be a quaternion algebra over $F$, and let $\mathcal{O} \subseteq B$ be an $R$-order. We say that $\mathcal{O}$ is Gorenstein if its codifferent is an invertible $R$-lattice in $B$, or equivalently $\operatorname{Hom}_{R}(\mathcal{O}, R)$ is projective as a left or right $\mathcal{O}$-module. Gorenstein orders were studied by Brzezinski [4, and they play a distinguished role in the taxonomy of quaternion orders - as Bass notes, Gorenstein rings are ubiquitous 2. Subsequent to this work, and given the importance of the Gorenstein condition, we say $\mathcal{O}$ is Bass if every $R$-superorder $\mathcal{O}^{\prime} \supseteq \mathcal{O}$ in $B$ is Gorenstein. As Bass himself showed [2], Bass orders enjoy good structural properties while also being quite general. A Bass order is Gorenstein, but not always conversely. Being Gorenstein or Bass is a local property over $R$, because invertibility is so.

On the other hand, we say that $\mathcal{O}$ is basic if there is a (commutative) quadratic $R$-algebra $S \subseteq \mathcal{O}$ such that $S$ is integrally closed in its total quotient ring $F S$. Basic orders were first introduced by Eichler $[8$ over $R=\mathbb{Z}$ (who called them primitive), and studied more generally by Hijikata-Pizer-Shemanske 12] (among their special orders), Brzezinski [5], and more recently by Jun [13. The embedded maximal quadratic $R$-algebra $S$ allows one to work explicitly with them, since a basic order $\mathcal{O}$ is locally free over $S$ of rank 2: for example, this facilitates the computation of the relevant quantities that arise in the trace formula [11. Locally, basic orders

Received by the editors March 14, 2019, and, in revised form, October 31, 2020.

The second author was supported by the Austrian Science Fund (FWF) project J4079-N32.

The third author was supported by an NSF CAREER Award (DMS-1151047) and a Simons Collaboration Grant (550029). 
also appear frequently: local Eichler orders are those that contain $R \times R$, and local Pizer (residually inert) orders [14, §2] are those orders in a division quaternion algebra that contain the valuation ring of an unramified quadratic extension. It is not immediate from the definition that being basic is a local property.

Results. The main result of this article is to show these two notions of Bass and basic coincide, in both local and global settings. We first consider the local case.

Theorem 1.1. Let $R$ be a discrete valuation ring $(D V R)$ and let $\mathcal{O}$ be a quaternion $R$-order. Then $\mathcal{O}$ is Bass if and only if $\mathcal{O}$ is basic.

Theorem 1.1 was proven by Brzezinski [5, Proposition 1.11] when $R$ is a complete DVR with char $R \neq 2$ and perfect residue field; the proof relies on a lengthy (but exhaustive) classification of Bass orders. Here, we present two essentially self-contained proofs that are uniform in the characteristic, one involving the manipulation of ternary quadratic forms and the second exploiting the structure of the radical.

Next, we turn to the global case.

Theorem 1.2. Let $R$ be a Dedekind domain whose field of fractions is a number field, and let $\mathcal{O}$ be a quaternion $R$-order. The following statements hold.

(a) $\mathcal{O}$ is basic if and only if the localization $\mathcal{O}_{(\mathfrak{p})}$ is basic for all primes $\mathfrak{p}$ of $R$.

(b) $\mathcal{O}$ is Bass if and only if $\mathcal{O}$ is basic.

In fact, we show that if $\mathcal{O}$ is Bass (equivalently, basic), then $\mathcal{O}$ contains infinitely many nonisomorphic quadratic $R$-algebras $S$ and moreover they can be taken to be free as $R$-modules (Corollary [7.6). Theorem 1.2 (b) over $R=\mathbb{Z}$ was proven by Eichler [8, Satz 8] using a somewhat different method.

We also prove the conclusions of Theorem 1.2 in a large number of cases in which $R$ is a Dedekind domain whose field of fractions is a global function field: see Theorem 7.5. (We lack in the function field case a sufficiently general local-global result on representations by ternary quadratic forms, see section 6. )

Returning to the local situation, if $R$ is a DVR then several equivalent characterizations of Bass orders are known [17, Proposition 24.5.3] and this list is further extended by our results. For the reader's convenience we give a comprehensive list.

Corollary 1.3. Let $R$ be a DVR with maximal ideal $\mathfrak{p}$, and let $\mathcal{O}$ be a quaternion $R$-order. Then the following are equivalent.

(i) $\mathcal{O}$ is a Bass order;

(ii) $\mathcal{O}$ and the radical idealizer $\mathcal{O}^{\natural}$ are Gorenstein;

(iii) The Jacobson radical $\operatorname{rad} \mathcal{O}$ is generated by two elements (as left, respectively, right ideal);

(iv) $\mathcal{O}$ is a basic order;

(v) Every $\mathcal{O}$-ideal is generated by two elements;

(vi) Every $\mathcal{O}$-lattice is isomorphic to a direct sum of $\mathcal{O}$-ideals; and

(vii) $\mathcal{O}$ is not of the form $\mathcal{O}=R+\mathfrak{p} I$ with $I$ an integral $R$-lattice.

The implications $(v) \Rightarrow(\mathrm{i}) \Rightarrow($ vi) hold more generally [17, Section 14.5]. The implication (vi) $\Rightarrow(\mathrm{v}$ ) holds only in specific settings; for quaternion orders it follows from work of Drozd-Kiričenko-Roŭter [7, Proposition 12.1, 12.5]. While we do not give another proof of this implication, we provide a direct proof for (i) $\Rightarrow(\mathrm{v})$. With the exception of statement (vi), we therefore give a full proof of the equivalences in Corollary 1.3 . 
Outline. The paper is organized as follows. After introducing some background in section 2, we prove Theorem 1.1 and Corollary 1.3 in sections 34. In the remaining sections, we prove Theorem 1.2 in section [5 we treat the case when strong approximation applies, in section [6, we treat definite orders over rings of integers in a number field, and we conclude the proof in section 7

\section{BACKGROUND}

In this section, we briefly review the necessary background on orders and quadratic forms. For a general reference, see Voight [17.

Properties of quaternion orders. Let $R$ be a Dedekind domain with $\operatorname{Frac}(R)=$ $F$. Let $B$ be a quaternion algebra over $F$ and let $\mathcal{O} \subseteq B$ be an $R$-order.

Definition 2.1. We say that $\mathcal{O}$ is Gorenstein if the codifferent

$$
\operatorname{codiff}(\mathcal{O}):=\{\alpha \in B: \operatorname{trd}(\alpha \mathcal{O}) \subseteq R\} \subseteq B
$$

is invertible, and we say $\mathcal{O}$ is Bass if every $R$-superorder $\mathcal{O}^{\prime} \supseteq \mathcal{O}$ is Gorenstein.

For more detail and further references, see Voight [17, Sections 24.2, 24.5]. Being Gorenstein is a local property - $\mathcal{O}$ is Gorenstein if and only if the localizations $\mathcal{O}_{(\mathfrak{p})}:=\mathcal{O} \otimes_{R} R_{(\mathfrak{p})}$ are Gorenstein for all primes $\mathfrak{p}$ of $R$ - so it follows that Bass is also a local property.

Definition 2.2. We say that $\mathcal{O}$ is basic if there is a (commutative) quadratic $R$ algebra $S \subseteq \mathcal{O}$ such that $S$ is integrally closed in its total quotient ring $F S$.

Remark 2.3. The term primitive is also used (in place of basic), but it is potentially confusing: we will see below that a primitive ternary quadratic form corresponds to a Gorenstein order, not a "primitive" order.

Local properties. Now suppose $R$ is a local Dedekind domain, i.e., $R$ is a discrete valuation ring (DVR) with maximal ideal $\mathfrak{p}$ and residue field $\kappa:=R / \mathfrak{p}$. The Jacobson radical of $\mathcal{O}$ is the intersection of all maximal left (or equivalently right) ideals of $\mathcal{O}$. The semisimple $\kappa$-algebra $\mathcal{O} / \operatorname{rad} \mathcal{O}$ is one of the following [17, 24.3.1]:

- $\mathcal{O} / \mathrm{rad} \mathcal{O}$ is a quaternion algebra (equivalently, $\mathcal{O}$ is maximal);

- $\mathcal{O} / \operatorname{rad} \mathcal{O} \simeq \kappa \times \kappa$, and we say that $\mathcal{O}$ is residually split (or Eichler);

- $\mathcal{O} / \operatorname{rad} \mathcal{O} \simeq \kappa$, and we say that $\mathcal{O}$ is residually ramified; or

- $\mathcal{O} / \operatorname{rad} \mathcal{O}$ is a separable quadratic field extension of $\kappa$ and we say that $\mathcal{O}$ is residually inert.

The radical idealizer of $\mathcal{O}$ is the left order $\mathcal{O}^{\natural}:=\mathcal{O}_{\mathrm{L}}(\operatorname{rad} \mathcal{O})$.

Ternary quadratic forms. Still with $R$ a DVR, we review the correspondence between quaternion orders and ternary quadratic forms (see also Voight [17, Chapters 5, 22] and [17, Remark 22.6.20] for a full history).

We define a similarity of two ternary quadratic forms $Q: R^{3} \rightarrow R$ and $Q^{\prime}: R^{3} \rightarrow R$ to be a pair $(f, u)$, where $f: R^{3} \rightarrow R^{3}$ is an $R$-module isomorphism and $u \in R^{\times}$is such that $Q^{\prime}(f(x))=u Q(x)$ for all $x \in \mathcal{O}$.

Proposition 2.4 (Gross-Lucianovic [10]). There is a discriminant-preserving bijection $\mathcal{O} \leftrightarrow Q(\mathcal{O})$ between quaternion $R$-orders up to isomorphism and nondegenerate ternary quadratic forms over $R$ up to similarity. Moreover, an $R$-order $\mathcal{O}$ is Gorenstein if and only if the corresponding quadratic form $Q(\mathcal{O})$ is primitive. 
We now briefly review the construction of the bijection in Proposition 2.4. Since $R$ is a PID, $\mathcal{O}$ is free of rank 4 as an $\mathcal{O}$-module. A good basis $1, i, j, k$ for an $R$-order $\mathcal{O}$ is an $R$-basis with a multiplication table of the form

$$
\begin{array}{ll}
i^{2}=u i-b c & j k=a \bar{i}=a(u-i) \\
j^{2}=v j-a c & k i=b \bar{j}=b(v-j) \\
k^{2}=w k-a b & i j=c \bar{k}=c(w-k)
\end{array}
$$

with $a, b, c, u, v, w \in R$. Every $R$-basis of $\mathcal{O}$ can be converted to a good basis in a direct manner. For all $x, y, z \in R$ and $\alpha=x i+y j+z k \in \mathcal{O}$, we find

$$
\begin{aligned}
& \operatorname{trd}(\alpha)=u x+v y+w z \\
& \operatorname{nrd}(\alpha)=b c x^{2}+a c y^{2}+a b z^{2}+(u v-c w) x y+(u w-b v) x z+(v w-a u) y z
\end{aligned}
$$

Associated to $\mathcal{O}$ and the good basis, we attach the ternary quadratic form $Q: R^{3} \rightarrow R$ defined by

$$
Q(x, y, z)=a x^{2}+b y^{2}+c z^{2}+u y z+v x z+w x y \in R[x, y, z] .
$$

The similarity class of $Q$ is well-defined on the isomorphism class of $\mathcal{O}$. Conversely, given a nondegenerate ternary quadratic form $Q: R^{3} \rightarrow R$, we associate to $Q$ its even Clifford algebra $\mathcal{O}=\operatorname{Clf}^{0}(Q)$, which is a quaternion $R$-order. A change of good basis of $\mathcal{O}$ induces a corresponding change of basis of $Q$, and conversely every such change of basis of $Q$ arises from a change of good basis of $\mathcal{O}$.

\section{LOCALLY BASS ORDERS ARE BASIC}

In this section, we give our first proof of Theorem 1.1. To this end, in this section and the next let $R$ be a DVR with fraction field $F:=\operatorname{Frac}(R)$ and maximal ideal $\mathfrak{p}=\pi R$. For $x, y \in R$, we write $\pi \mid x, y$ for $\pi \mid x$ and $\pi \mid y$.

Let $B$ be a quaternion algebra over $F$ and $\mathcal{O} \subseteq B$ an $R$-order. According to the following remark, we could work equivalently in the completion of $R$.

Remark 3.1. The order $\mathcal{O}$ is basic (or Bass) if and only if its completion is basic (or Bass). Indeed, invertibility and maximality can be checked in the completion.

We choose a good $R$-basis $1, i, j, k$ for $\mathcal{O}$ and let $Q$ be the ternary quadratic form over $R$ associated to $\mathcal{O}$ with respect to this basis, as in (2.7).

Lemma 3.2. The order $\mathcal{O}$ is not basic if and only if for every $\alpha \in \mathcal{O}$ there exists $r \in R$ such that $\pi \mid \operatorname{trd}(\alpha-r)$ and $\pi^{2} \mid \operatorname{nrd}(\alpha-r)$.

Proof. Let $\alpha \in \mathcal{O}$ and consider the $R$-algebra $R[\alpha]=R+R \alpha$. Then $R[\alpha]$ fails to be integrally closed if and only if there exists $\beta \in F[\alpha]$, integral over $R$, such that $\beta \notin R[\alpha]$; this holds if and only if there exists $r \in R$ such that $\beta=\pi^{-1}(\alpha-r)$ is integral over $R$, which is equivalent to $\operatorname{trd}(\beta)=\pi^{-1} \operatorname{trd}(\alpha-r) \in R$ and $\operatorname{nrd}(\beta)=$ $\pi^{-2} \operatorname{nrd}(\alpha-r) \in R$, as claimed.

A slight reformulation gives a local version of the result of Eichler [8, Satz 8]. Recall that a semi-order $I \subseteq B$ is an integral $R$-lattice with $1 \in I$ [17, Section 16.6]. Basic semi-orders are defined analogously to basic orders.

Lemma 3.3. A semi-order $I$ is not basic if and only if it is of the form $I=R+\mathfrak{p} J$ with $J \subseteq B$ an integral $R$-lattice. 
Proof. As in the previous lemma, if $I=R+\mathfrak{p} J$, then $I$ is not basic. Conversely, if $I$ is not basic, each $\alpha \in I$ is of the form $\alpha-r=\pi \beta$ with an integral $\beta$. Take $J$ to be the $R$-lattice generated by all these $\beta$.

As an application of Lemma 3.2, we prove one implication in Theorem 1.1

Proposition 3.4. If $\mathcal{O}$ is basic, then $\mathcal{O}$ is Bass.

Proof. Suppose $\mathcal{O}$ is basic. Then every $R$-superorder $\mathcal{O}^{\prime} \supseteq \mathcal{O}$ is also basic. So to show that $\mathcal{O}$ is Bass, we may show that $\mathcal{O}$ is Gorenstein. To do so, we prove the contrapositive. Suppose that $\mathcal{O}$ is not Gorenstein. Then the quadratic form $Q$ associated to $\mathcal{O}$ has all coefficients $a, b, c, u, v, w \in \mathfrak{p}$. From (2.6), we see that for all $\alpha=x i+y j+z k \in \mathcal{O}$ we have $\pi \mid \operatorname{trd}(\alpha)$ and $\pi^{2} \mid \operatorname{nrd}(\alpha)$. Therefore $\mathcal{O}$ is not basic by Lemma 3.2

Lemma 3.5. If $\mathcal{O}$ is maximal, residually inert, or residually split, then $\mathcal{O}$ is basic and Bass.

Proof. By the previous proposition it suffices to show that $\mathcal{O}$ is basic. In each case, $\mathcal{O} / \mathrm{rad} \mathcal{O}$ contains a separable quadratic algebra over $R / \mathfrak{p}$ which lifts to a valuation ring in $\mathcal{O}$. See also Voight [17, 24.5.2, Proposition 24.5.5].

Remark 3.6. It is not always possible to embed an integrally closed quadratic order that is a domain into a residually split (Eichler) order; this justifies the (more general) definition of basic orders allowing nondomains such as $R \times R$.

Lemma 3.7. Suppose $\mathcal{O}$ is Gorenstein with associated quadratic form $Q$ in a good basis as in (2.7) and that $\mathcal{O}$ is not basic. Then the following statements hold.

(a) If $\pi \mid 2$ in $R$, then $\pi \mid u, v, w$.

(b) Suppose that $\pi \mid u, v, w$. Let $s \in\{a, b, c\}$ and suppose $\pi \mid s$. Then $\pi^{2} \mid s$.

Proof. For (a), to show that $\pi \mid u$, by Lemma 3.2 there exists $r \in R$ such that $\pi \mid \operatorname{trd}(i-r)=u-2 r$; since $\pi \mid 2$, we have $\pi \mid u$. Similarly, arguing with $j, k$ we have $\pi \mid v, w$. For (b), without loss of generality we suppose $s=a$ and $b \in R^{\times}$. By Lemma 3.2

$$
\pi^{2} \mid \operatorname{nrd}(k-r)=\operatorname{nrd}(k)-r \operatorname{trd}(k)+r^{2}=a b-r w+r^{2} .
$$

But $\pi \mid a, w$, so $\pi \mid r^{2}$. Thus $\pi \mid r$, so $\pi^{2} \mid r w, r^{2}$, so $\pi^{2} \mid a b$; since $b \in R^{\times}$, we get $\pi^{2} \mid a$.

Lemma 3.9. Suppose $\mathcal{O}$ is Gorenstein, not basic, and residually ramified. Then there exists a good basis of $\mathcal{O}$ such that the associated quadratic form is given by

$$
Q(x, y, z)=a x^{2}+b y^{2}+c z^{2}+u y z+w x y,
$$

with $\pi \mid u, w$ and $\pi^{2} \mid c$ and one of the following conditions holds:

(i) $a \in R^{\times}$and $\pi^{2} \mid b$; or

(ii) $\pi^{2} \mid a$ and $b \in R^{\times}$and $w=0$.

Proof. As explained in section 2, a change of good basis of $\mathcal{O}$ corresponds to a change of basis for $Q$, so we work with the latter. By a standard "normal form" argument (see e.g. Voight [16, Proposition 3.10]), there exists a basis $e_{11}, e_{12}, e_{13}$ such that $Q$ becomes

$$
Q_{1}(x, y, z)=a_{1} x^{2}+b_{1} y^{2}+c_{1} z^{2}+u_{1} y z
$$


with $a_{1}, b_{1}, c_{1}, u_{1} \in R$ and not all in $\mathfrak{p}$, and $u_{1}=0$ if $2 \in R^{\times}$. Let $1, i_{1}, j_{1}, k_{1}$ be the corresponding good basis for $\mathcal{O}$.

We modify this basis further to obtain the desired divisibility, as follows. First, suppose that $2 \in R^{\times}$. Then $u_{1}=0$. Swapping basis vectors, we obtain the diagonal quadratic form $Q_{2}(x, y, z)=a_{2} x^{2}+b_{2} y^{2}+c_{2} z^{2}$ with $a_{2} \in R^{\times}$. If $b_{2} \in R^{\times}$, then $k \in \mathcal{O}$ satisfies $k^{2}=-a_{2} b_{2} \in R^{\times}$so $\mathcal{O}$ is not residually ramified, a contradiction, so we must have $\pi \mid b_{2}$ and by symmetry $\pi \mid c_{2}$. By Lemma 3.7, we get $\pi^{2} \mid b_{2}, c_{2}$, and we are in case (i) (which becomes case (ii) after a basis swap).

Second, suppose that $2 \notin R^{\times}$, so $\pi \mid 2$. By Lemma 3.7(a), we have $\pi \mid u_{1}$. If $\pi \mid c_{1}$, we keep the basis unchanged and pass all subscripts 1 to 2 . If $\pi \mid b_{1}$, we take $e_{21}, e_{22}, e_{23}:=e_{11}, e_{13}, e_{12}$ (swapping second and third basis elements); in this basis, we obtain the quadratic form

$$
Q_{2}(x, y, z)=a_{2} x^{2}+b_{2} y^{2}+c_{2} z^{2}+u_{2} y z
$$

with $a_{2}=a_{1}, b_{2}=c_{1}, c_{2}=b_{1}$, and $u_{2}=u_{1}$, with $\pi \mid c_{2}$. Otherwise, suppose $b_{1}, c_{1} \in R^{\times}$. Since $\mathcal{O}$ is residually ramified, we have $\mathcal{O} / \operatorname{rad} \mathcal{O} \simeq R / \mathfrak{p}$. Moreover $i_{1}^{2}=u_{1} i_{1}-b_{1} c_{1}$. Reducing modulo $\mathfrak{p}$, we conclude that $-b_{1} c_{1} \in(R / \mathfrak{p})^{\times 2}$, so there exists $s_{1} \in R$ such that $s_{1}^{2} \equiv-c_{1} b_{1}^{-1}(\bmod \mathfrak{p})$. We take the new basis $e_{21}, e_{22}, e_{23}:=$ $e_{11}, e_{12}, e_{13}+s_{1} e_{12}$. In this basis, we obtain (3.11) where now

$$
a_{2}=a_{1}, b_{2}=b_{1}, c_{2}=c_{1}+s_{1} u_{1}+s_{1}^{2} b_{1} \text {, and } u_{2}=u_{1}+2 s_{1} b_{1} .
$$

Since $\pi \mid u_{1}$ and $\pi \mid\left(c_{1}+s_{1}^{2} b_{1}\right)$, we have $\pi \mid c_{2}$. In all cases, we have $\pi \mid c_{2}$. By Lemma 3.7, we immediately upgrade to $\pi^{2} \mid c_{2}$. Finally, since $\mathcal{O}$ is Gorenstein, either $\pi \mid a_{2}$ and then $b_{2} \in R^{\times}$and $\pi^{2} \mid a_{2}$ as in case (ii), or we have $a_{2} \in R^{\times}$.

To finish, we suppose that $a_{2} \in R^{\times}$and we make one final change of basis to get us into case (i). As in the previous paragraph, we have $k_{2}^{2}=-a_{2} b_{2}$, so there exists $s_{2} \in R$ such that $s_{2}^{2} \equiv-b_{2} a_{2}^{-1}(\bmod \mathfrak{p})$. We take the new basis $e_{31}, e_{32}, e_{33}:=e_{21}, e_{22}+s_{2} e_{21}, e_{23}$, giving the quadratic form $Q_{3}(x, y, z)=a_{3} x^{2}+$ $b_{3} y^{2}+c_{3} z^{2}+u_{3} y z+w_{3} x y$ and

$$
a_{3}=a_{2}, b_{3}=b_{2}+a_{2} s_{2}^{2}, c_{3}=c_{2}, u_{3}=u_{2}, \text { and } w_{3}=2 a_{2} s_{2} .
$$

Now $\pi \mid b_{3}$ by construction, and $\pi \mid u_{3}, w_{3}$ so $\pi^{2} \mid b_{3}$ and we get to case (i).

We now prove Theorem 1.1 .

Theorem 3.12. The order $\mathcal{O}$ is Bass if and only if $\mathcal{O}$ is basic.

Proof. We proved $(\Leftarrow)$ in Proposition 3.4. We prove $(\Rightarrow)$ by the contrapositive: we suppose that $\mathcal{O}$ is not basic and show $\mathcal{O}$ is not Bass by exhibiting a $R$-superorder $\mathcal{O}^{\prime} \supseteq \mathcal{O}$ that is not Gorenstein. If $\mathcal{O}$ is not Gorenstein then it is not Bass, so we are done. Suppose then that $\mathcal{O}$ is Gorenstein. By Lemma 3.5 we must have $\mathcal{O}$ residually ramified. Then by Lemma 3.9, there exists a good basis for $\mathcal{O}$ such that the corresponding quadratic form satisfies either (i) or (ii) from that lemma.

We begin with case (i). We first claim that $\pi^{2} \mid u$. By Lemma 3.2, there exists $r$ such that $\pi^{2} \mid \operatorname{nrd}(j+k-r)=a c+a b-a u-r w+r^{2} ;$ since $\pi \mid b, c, u, w$ we conclude $\pi \mid r$; then $\pi^{2} \mid b, c, r^{2}, r w$ implies $\pi^{2} \mid a u$, and since $a \in R^{\times}$we get $\pi^{2} \mid u$. This gives us a (minimal) non-Gorenstein superorder, as follows. Let $i^{\prime}:=\pi^{-1} i$ and let $\mathcal{O}^{\prime}:=R+R i^{\prime}+R j+R k$. Then $\mathcal{O}^{\prime} \supseteq \mathcal{O}$ and $\mathcal{O}^{\prime}$ has the following multiplication table, with coefficients

$$
a^{\prime}:=\pi a, b^{\prime}:=\pi^{-1} b, c^{\prime}:=\pi^{-1} c, u^{\prime}:=\pi^{-1} u, w^{\prime}:=w
$$


in $R$ :

$$
\begin{aligned}
\left(i^{\prime}\right)^{2} & =\pi^{-2}(u i-b c)=u^{\prime} i^{\prime}-b^{\prime} c^{\prime} & & j k=a \bar{i}=a^{\prime} \bar{i}^{\prime} \\
j^{2} & =-a c=-a^{\prime} c^{\prime} & k i^{\prime} & =\pi^{-1} b \bar{j}=b^{\prime} \bar{j} \\
k^{2} & =w k-a b=w^{\prime} k-a^{\prime} b^{\prime} & i^{\prime} j & =\pi^{-1} c \bar{k}=c^{\prime} \bar{k} .
\end{aligned}
$$

Thus $\mathcal{O}^{\prime}$ is an $R$-order with $Q^{\prime}(x, y, z)=a^{\prime} x^{2}+b^{\prime} y^{2}+c^{\prime} z^{2}+u^{\prime} y z+w^{\prime} x y$, all of whose coefficients are divisible by $\pi$. We conclude $\mathcal{O}^{\prime}$ is not Gorenstein and so $\mathcal{O}$ is not Bass.

Case (ii) follows similarly, taking instead $j^{\prime}:=\pi^{-1} j$ and $\mathcal{O}^{\prime}:=R+R i+R j^{\prime}+R k$, with associated quadratic form $Q^{\prime}(x, y, z)=a^{\prime} x^{2}+b^{\prime} y^{2}+c^{\prime} z^{2}+u^{\prime} y z$ satisfying $a^{\prime}=\pi^{-1} a, b^{\prime}=\pi b, c^{\prime}=\pi^{-1} c, u^{\prime}=u$, all of which are divisible by $\pi$.

Remark 3.14. If $\mathcal{O}$ is a Gorenstein order that is neither residually split nor maximal, the radical idealizer $\mathcal{O}^{\natural}=\mathcal{O}_{L}(\operatorname{rad} \mathcal{O})=\mathcal{O}_{R}(\operatorname{rad} \mathcal{O})$ is the unique minimal superorder by [17, Proposition 24.4.12]. In the previous proof $\left[\mathcal{O}^{\prime}: \mathcal{O}\right]_{\mathfrak{p}}=\mathfrak{p}$, and hence necessarily $\mathcal{O}^{\natural}=\mathcal{O}^{\prime}$. We have therefore proved that if $\mathcal{O}$ and $\mathcal{O}^{\natural}$ are both Gorenstein, then $\mathcal{O}$ is basic. We return to this in the next section.

Remark 3.15. When $2 \in R^{\times}$, the argument for Theorem 1.1] is quite simple [17, Proposition 24.5.8]: diagonalizing up to similarity, the ternary quadratic form associated to a Gorenstein order $\mathcal{O}$ is $x^{2}+b y^{2}+c z^{2}$ with $v(b) \leq v(c)$, and $\mathcal{O}$ is Bass if and only if $v(b) \leq 1$.

\section{A SECOND PROOF FOR LOCAL BASS ORDERS BEING BASIC}

In this section, we given a second proof of (the hard direction of) Theorem 1.1. We retain our notation from the previous section; in particular $R$ is a discrete valuation ring with maximal ideal $\mathfrak{p}=\pi R$.

By classification, we see that a quaternion $R$-order $\mathcal{O}$ is a local ring (has a unique maximal left [right] ideal, necessarily equal to its Jacobson radical $\operatorname{rad} \mathcal{O}$ ) if and only if $\mathcal{O}$ is neither maximal nor residually split.

Lemma 4.1. Suppose that $\mathcal{O}$ is a local ring. Let $\alpha \in \operatorname{rad} \mathcal{O}$. Then the following statements hold.

(a) We have $\pi \mid \operatorname{trd}(\alpha), \operatorname{nrd}(\alpha)$ and $\alpha^{2} \in \mathfrak{p O}$.

(b) If $\mathcal{O}$ is not basic, then $\pi^{2} \mid \operatorname{nrd}(\alpha)$ and $\alpha^{2} \in \mathfrak{p} \operatorname{rad} \mathcal{O}$.

Proof. Since $\mathcal{O} / \mathfrak{p O}$ is Artinian, $(\operatorname{rad} \mathcal{O}) / \mathfrak{p O}$ is nilpotent, so there exists $r \in \mathbb{Z}_{\geq 1}$ such that $\alpha^{r} \equiv 0(\bmod \mathfrak{p O})$. Thus the image of $\alpha$ in the $R / \mathfrak{p}$-algebra has reduced characteristic polynomial $x^{2}$, so $\alpha^{2} \in \mathfrak{p O}$ and $\operatorname{trd}(\alpha), \operatorname{nrd}(\alpha) \equiv 0(\bmod \mathfrak{p})$, proving (a). Since $\alpha$ satisfies its reduced characteristic polynomial $f(x)=x^{2}-\operatorname{trd}(\alpha) x+$ $\operatorname{nrd}(\alpha) \in R[x]$, if $\pi^{2} \nmid \operatorname{nrd}(\alpha)$, then $f(x)$ is an Eisenstein polynomial so $R[\alpha]$ is a DVR and in particular integrally closed, contradicting that $\mathcal{O}$ is not basic and proving the first part of (b). Finally, $\operatorname{nrd}(\alpha) \in \mathfrak{p}^{2} \subseteq \mathfrak{p} \operatorname{rad} \mathcal{O}$ so $\alpha^{2}=\operatorname{trd}(\alpha) \alpha-$ $\operatorname{nrd}(\alpha) \in \mathfrak{p} \operatorname{rad} \mathcal{O}$.

Lemma 4.2. Let $A$ be a local Artinian $R$-algebra with $R / \mathfrak{p} \simeq A / \operatorname{rad} A$ via $R \rightarrow A$. If $y_{1}, \ldots, y_{n}$ generate $\operatorname{rad} A$ as ideal of $A$, then they generate $A$ as $R$-algebra.

Proof. Let $J=\operatorname{rad} A$. Since $A$ is Artinian, there exists $m \geq 0$ with $J^{m}=0$. For $l \in[1, m]$, let $Z_{l}=\left\{y_{\nu_{1}} \cdots y_{\nu_{l}}: \nu_{1}, \ldots, \nu_{l} \in 1, \ldots, n\right\}$. Since $R / \mathfrak{p} \simeq A / J$ 
is commutative, it is easily seen that $Z_{l}$ generates $J^{l} / J^{l+1}$ as $R / \mathfrak{p}$-module. Using $A \supseteq J \supseteq \cdots \supseteq J^{m}=0$, we see that $\{1\} \cup Z_{1} \cup \cdots \cup Z_{l}$ generates $A$ as $R$-module.

Theorem 4.3. Let $\mathcal{O}$ be a residually ramified Bass $R$-order and suppose that $\operatorname{rad} \mathcal{O}$ is generated by two elements as left [right] ideal. Then $\mathcal{O}$ is basic.

Proof. Let $J=\operatorname{rad} \mathcal{O}$, and let $\alpha_{1}, \alpha_{2}$ generate $J$ as left ideal (the other case being symmetric). Their images generate $J / J^{2}$ over $\mathcal{O} / J \simeq R / \mathfrak{p}$, so $\operatorname{dim}_{R / \mathfrak{p}} J / J^{2} \leq 2$.

Suppose to the contrary that $\mathcal{O}$ is not basic. Observe $\pi J \subseteq J^{2}$ since $\pi \mathcal{O} \subseteq J$. Lemma 4.1 implies $\alpha_{1}^{2}, \alpha_{2}^{2},\left(\alpha_{1}+\alpha_{2}\right)^{2} \in \pi J$. Thus $0 \equiv\left(\alpha_{1}+\alpha_{2}\right)^{2} \equiv \alpha_{1} \alpha_{2}+\alpha_{2} \alpha_{1}$ $(\bmod \pi J)$. By Lemma 4.2 the elements $\alpha_{1}+\pi J, \alpha_{2}+\pi J$ generate $\mathcal{O} / \pi J$ as $R$ algebra. Since they anticommute, we see that they are normal elements in $\mathcal{O} / \pi J$. It follows that $J^{2} / \pi J$ is generated by $\alpha_{1} \alpha_{2}+\pi J$ as $\mathcal{O} / \pi J$-module.

Again using that $\alpha_{1}+\pi J$ and $\alpha_{2}+\pi J$ anticommute, we have $\alpha_{1}\left(\alpha_{1} \alpha_{2}\right) \equiv 0 \equiv$ $\alpha_{2}\left(\alpha_{1} \alpha_{2}\right)(\bmod \pi J)$. This implies that $J^{2} / \pi J$ is in fact generated by $\alpha_{1} \alpha_{2}+\pi J$ as $\mathcal{O} / J$-module, and hence as $R / \mathfrak{p}$-vector space.

Let $\lambda(M)$ be the length of an $\mathcal{O}$-module $M$. Since $\operatorname{dim}_{R / \mathfrak{p}} J / J^{2} \leq 2$ we have $\lambda\left(\mathcal{O} / J^{2}\right) \leq 3$. Because $O$ is residually ramified and $J / \pi \mathcal{O} \simeq \pi J / \pi^{2} \mathcal{O}$ we find $\lambda\left(\pi J / \pi^{2} \mathcal{O}\right)=3$. Now

$$
8=\lambda\left(\mathcal{O} / \pi^{2} \mathcal{O}\right)=\lambda(\mathcal{O} / \pi J)+\lambda\left(\pi J / \pi^{2} \mathcal{O}\right)=\lambda(\mathcal{O} / \pi J)+3
$$

yields $\lambda(\mathcal{O} / \pi J)=5$. But $\lambda\left(J^{2} / \pi J\right)=1$ gives $\lambda\left(\mathcal{O} / J^{2}\right)=4$, a contradiction.

The previous theorem together with the characterization of Bass orders 17. Proposition 24.5.3] implies that every (residually ramified) Bass order is basic. Alternatively, it is easy to see directly that the assumption of Theorem 4.3 holds for Bass orders, as the next proposition shows.

Proposition 4.5. If $\mathcal{O}$ and $\mathcal{O}^{\natural}$ are Gorenstein $R$-orders, then $\operatorname{rad} \mathcal{O}$ is generated by two elements (as a left, respectively, right $\mathcal{O}$-ideal).

Proof. If $\mathcal{O}$ is hereditary, then $\operatorname{rad} \mathcal{O}$ is principal 17, Main Theorems 21.1.4 and 16.6.1]. If $\mathcal{O}$ is Eichler, it is easily seen from an explicit description of $\mathcal{O}$ that $\operatorname{rad} \mathcal{O}$ is generated by two elements [17, 23.4.15]. We thus suppose that $\mathcal{O}$ is a local ring.

Let $J=\operatorname{rad} \mathcal{O}$. Then $\mathcal{O}^{\natural}=\left(J \mathcal{O}^{\#}\right)^{\#}$ with $\mathcal{O}^{\#}=\mathcal{O} \alpha$ for some $\alpha \in B^{\times}[17$, Proposition 24.4.12]. (using that $\mathcal{O}$ is Gorenstein). Since $J \mathcal{O}^{\#}$ is the unique maximal left [right] $\mathcal{O}$-submodule of $\mathcal{O}^{\#}$ by the proof of the same proposition, dualizing implies that there is no right [left] $\mathcal{O}$-module properly between $\mathcal{O}$ and $\mathcal{O}^{\natural}$ [17, Section 15.5]. Hence $\mathcal{O}^{\natural} / \mathcal{O}$ is a cyclic right [left] $\mathcal{O}$-module. So $\mathcal{O}^{\natural}=\mathcal{O}+\beta \mathcal{O}=\mathcal{O}+\mathcal{O} \beta^{\prime}$ with $\beta, \beta^{\prime} \in \mathcal{O}^{\natural}$. Since $\mathcal{O}^{\natural}$ is also Gorenstein and $O_{\mathrm{L}}(J)=\mathcal{O}^{\natural}$, the ideal $J$ is invertible and hence principal [17, Proposition 24.2.3 and Main Theorem 16.6.1]. So $J=\gamma \mathcal{O}^{\natural}=\mathcal{O}^{\natural} \gamma^{\prime}$. Altogether $J=\gamma \mathcal{O}+\gamma \beta \mathcal{O}=\mathcal{O} \gamma^{\prime}+\mathcal{O} \gamma^{\prime} \beta^{\prime}$.

We now characterize local Bass orders.

Proof of Corollary 1.3, (i) $\Rightarrow$ (ii) by definition; (ii) $\Rightarrow$ (iii) is Proposition 4.5, (iii) $\Rightarrow$ (iv) by Theorem 4.3 for residually ramified orders, in any other case $\mathcal{O}$ is basic without any assumption on $\operatorname{rad} \mathcal{O}$ by Lemma 3.5. Propositon 3.4 shows (iv) $\Rightarrow$ (i).

(iv) $\Rightarrow(\mathrm{v})$ Let $S$ be a maximal order of a $F$-quadratic algebra contained in $\mathcal{O}$. Any $\mathcal{O}$-ideal $I$ is an $S$-lattice of rank 2 . Since $S$ is local, $I$ is a free $S$-lattice of rank two. Thus $I$ is generated by two elements over $S$ and also over $\mathcal{O}$ (as left or right ideal). (v) $\Rightarrow$ (iii) is trivial. 
(i) $\Leftrightarrow$ (vi) holds [17, Proposition 24.5.3]. The implications (v) $\Rightarrow$ (i) $\Rightarrow$ (vi) hold in large generality, whereas $(\mathrm{vi}) \Rightarrow(\mathrm{v})$ for quaternion orders is a result of DrozdKiričenko-Roiter. Finally, (iv) $\Leftrightarrow$ (vii) follows from Lemma 3.3

\section{BASIC ORDERS UNDER STRONG APPROXIMATION}

In this section, we prove Theorem 1.2 when strong approximation applies. We start by showing that basic is a local property, i.e., an $R$-order $\mathcal{O}$ is basic if and only if its localization at every nonzero prime $\mathfrak{p}$ of $R$ is basic.

Setup. Moving now from the local to the global setting, we use the following notation. Let $F$ be a global field and let $R=R_{(T)} \subseteq F$ be the ring of $T$-integers for a nonempty finite set $T$ of places of $F$ containing the archimedean places. Let $B$ be a quaternion algebra over $F$, and let $\mathcal{O} \subseteq B$ be an $R$-order. For a prime $\mathfrak{p} \subseteq R$, define the normalized valuation $v_{\mathfrak{p}}$ with valuation ring $R_{(\mathfrak{p})} \subseteq F$, and similarly define $\mathcal{O}_{(\mathfrak{p})}:=\mathcal{O} \otimes_{R} R_{(\mathfrak{p})} \subseteq B$.

Building global quadratic orders. Using discriminants, we combine local (embedded) quadratic orders to construct a candidate global quadratic order which we may try to embed in $\mathcal{O}$. Recall that free quadratic $R$-orders are, via the discriminant, in bijection with elements $d \in R / R^{\times 2}$ that are squares in $R / 4 R$.

Lemma 5.1. Suppose that $\mathcal{O}_{(\mathfrak{p})}$ is basic for all $\mathfrak{p}$. Then there exist infinitely many $d \in R / R^{\times 2}$, corresponding to integrally closed quadratic $R$-orders $S$ (up to isomorphism), such that $S_{(\mathfrak{p})}$ embeds in $\mathcal{O}_{(\mathfrak{p})}$.

Proof. For each $\mathfrak{p}$, let $S(\mathfrak{p})$ be an integrally closed quadratic $R_{(\mathfrak{p})}$-order in $\mathcal{O}_{(\mathfrak{p})}$ and let $d(\mathfrak{p}):=\operatorname{disc}(S(\mathfrak{p}))$. For each $\mathfrak{p} \mid \operatorname{discrd}(\mathcal{O})$, let $e_{\mathfrak{p}}:=v_{\mathfrak{p}}(d(\mathfrak{p}))$. If $\mathfrak{p} \nmid 2 R$, then $e_{\mathfrak{p}} \leq 1$ by maximality of $S(\mathfrak{p})$. Define

$$
\mathfrak{d}:=\prod_{\mathfrak{p} \mid \operatorname{discrd}(\mathcal{O})} \mathfrak{p}^{e_{\mathfrak{p}}} .
$$

By the Chebotarev density theorem applied to the Hilbert class field of $F$, there exist infinitely many prime ideals $\mathfrak{q} \subseteq R$ such that $\mathfrak{q} \nmid 2 \operatorname{discrd}(\mathcal{O})$ and $\mathfrak{d} \mathfrak{q}=d^{\prime} R$ is principal. Let

$$
t_{\mathfrak{p}}:= \begin{cases}1, & \text { if } \mathfrak{p} \nmid 2 R \\ \max \left\{2 v_{\mathfrak{p}}(2)+1, e_{\mathfrak{p}}\right\}, & \text { if } \mathfrak{p} \mid 2 R\end{cases}
$$

and let

$$
\mathfrak{n}:=\prod_{\mathfrak{p} \mid 2 \operatorname{discrd}(\mathcal{O})} \mathfrak{p}^{t_{\mathfrak{p}}} .
$$

By the Chinese Remainder Theorem, there is an element $a \in R$ such that $a \equiv$ $d(\mathfrak{p})\left(d^{\prime}\right)^{-1}\left(\bmod \mathfrak{p}^{t_{\mathfrak{p}}}\right)$ for each $\mathfrak{p} \mid 2 \operatorname{discrd}(\mathcal{O})$. By the Chebotarev density theorem applied to the ray class field of $F$ of conductor $\mathfrak{n}$, there exist infinitely many prime elements $\pi \in R$ such that $\pi \equiv a(\bmod \mathfrak{n})$.

Define $d:=d^{\prime} \pi$, so $d R=\mathfrak{d} \mathfrak{q} \pi$. Then for $\mathfrak{p} \mid 2 \operatorname{discrd}(\mathcal{O})$, we have $d=u_{\mathfrak{p}} d(\mathfrak{p})$, where $u_{\mathfrak{p}}=d^{\prime} \pi d(\mathfrak{p})^{-1} \equiv 1(\bmod \mathfrak{n})$. Because $4 \mid \mathfrak{n}$, the element $d$ is a square in $R / 4 R$. Let $S$ be the (free) quadratic $R$-order of discriminant $d$. Then $S_{(\mathfrak{p})} \simeq S(\mathfrak{p})$ for $\mathfrak{p} \mid 2 \operatorname{discrd}(\mathcal{O})$, which is integrally closed. For $\mathfrak{p} \nmid 2 \operatorname{discrd}(\mathcal{O})$, we have $S_{(\mathfrak{p})} \hookrightarrow$ $\mathcal{O}_{(\mathfrak{p})} \simeq \mathrm{M}_{2}\left(R_{(\mathfrak{p})}\right)$, and $S_{(\mathfrak{p})}$ is integrally closed because $v_{\mathfrak{p}}(d) \leq 1$. Therefore, $S_{(\mathfrak{p})}$ is integrally closed for each prime $\mathfrak{p}$, so $S$ is integrally closed. Since there were infinitely many choices for primes $\mathfrak{q}$ and $\pi$, the same is true for $S$. 
Selectivity conditions. We must now show that we can choose $S$ in Lemma 5.1 such that $S \hookrightarrow \mathcal{O}$. To reach this conclusion, we now invoke the hypothesis that $B$ is $T$-indefinite, so that strong approximation [17, Chapter 28] applies.

Lemma 5.4. Suppose that $B$ is T-indefinite. Then for all but finitely many integrally closed quadratic $R$-orders $S$ we have $S \hookrightarrow \mathcal{O}$ if and only if $S_{(\mathfrak{p})} \hookrightarrow \mathcal{O}_{(\mathfrak{p})}$ for all primes $\mathfrak{p}$ of $R$.

Proof. Let $\mathscr{L}$ be the set of integrally closed quadratic orders $S$ (up to isomorphism) such that $S_{(\mathfrak{p})} \hookrightarrow \mathcal{O}_{(\mathfrak{p})}$ for all $\mathfrak{p}$. We refer to Voight [17, Main Theorem 31.1.7]: under the hypothesis that $B$ is $T$-indefinite, there exists a finite extension $L:=H_{G N(\mathcal{O})} \supseteq$ $F$ with the property that $S \in \mathscr{L}$ embeds in $\mathcal{O}$ whenever $K:=\operatorname{Frac}(S)$ is not a subfield of $L$. As there are only finitely many subfields $K \subseteq L$, only finitely many $S \in \mathscr{L}$ will not embed in $\mathcal{O}$.

Lemma 5.5. Suppose that $B$ is T-indefinite, and suppose $\mathcal{O}_{(\mathfrak{p})}$ is basic for every prime $\mathfrak{p}$ of $R$. Then $\mathcal{O}$ contains infinitely many nonisomorphic integrally closed quadratic R-orders.

Proof. Suppose that $\mathcal{O}_{(\mathfrak{p})}$ is basic for every prime $\mathfrak{p}$ of $R$. Then, $\mathcal{O}_{(\mathfrak{p})}$ contains a maximal commutative $R_{(\mathfrak{p})}$-order for every prime $\mathfrak{p}$. By Lemma 5.1, there exist infinitely many $d \in R / R^{\times 2}$ such that the corresponding quadratic order $S_{d}$ is integrally closed and $\left(S_{d}\right)_{(\mathfrak{p})} \hookrightarrow \mathcal{O}_{(\mathfrak{p})}$ for all $\mathfrak{p}$. For all but finitely many such choices of $d$, we have an embedding $S_{d} \hookrightarrow \mathcal{O}$.

Proof of theorem. With these lemmas in hand, we now prove Theorem 1.2 under the hypothesis that $B$ is $T$-indefinite and $\# T<\infty$.

Proof of Theorem 1.2, $B$ is T-indefinite and $\# T<\infty$. First, part (a). If $\mathcal{O}_{(\mathfrak{p})}$ is basic for every prime $\mathfrak{p}$ of $R$, then $\mathcal{O}$ contains an integrally closed quadratic $R$-order by Lemma 5.5. Conversely, if $\mathcal{O}$ is basic, then it contains a maximal commutative $R$-order $S$. Then, the localization $S_{(\mathfrak{p})}:=S \otimes R_{(\mathfrak{p})}$ at every prime $\mathfrak{p}$ is a maximal $R_{(\mathfrak{p})}$-order in $\mathcal{O}_{(\mathfrak{p})}$ by the local-global dictionary for lattices, so $\mathcal{O}_{(\mathfrak{p})}$ is basic for every prime $\mathfrak{p}$ of $R$. Being Bass is a local property, and local orders are basic if and only if they are Bass by Theorem 3.12 , so (b) follows from (a).

This proof gives in fact a bit more.

Corollary 5.6. Suppose that $B$ is $T$-indefinite. If $\mathcal{O}$ is basic, then $\mathcal{O}$ contains infinitely many nonisomorphic integrally closed quadratic $R$-orders.

Proof. Combine Theorem 1.2(a) with Lemma 5.5.

\section{BASIC ORDERS AND DEFINITE TERnARY THETA SERIES}

In this section, we finish the proof of Theorem 1.2 in the remaining case of a $T$-definite quaternion algebra under some hypotheses. For this purpose, we replace the application of strong approximation with a statement on representations of ternary quadratic forms. 
Ternary representations. As above, let $F$ be a global field, let $T$ be a nonempty finite set of places of $F$ containing the archimedean places, and let $R=R_{(T)} \subseteq F$ be the ring of $T$-integers in $F$. For nonzero $a \in R$, we write $\mathrm{N}(a):=\#(R / a R)$ for the absolute norm of $a$.

Conjecture 6.1 (Ternary representation). Let $Q: M \rightarrow R$ be a nondegenerate ternary quadratic form over $R=R_{(T)}$ such that $Q_{v}$ is anisotropic for all $v \in T$. Then there exists $c_{Q} \in \mathbb{R}_{>0}$ such that every squarefree $a \in R$ with $\mathrm{N}(a) \geq c_{Q}$ is represented by $Q$ if and only if a is represented by the completion $Q_{v}$ for all places $v$ of $F$.

For further reading, see Schulze-Pillot [15] and Cogdell [6]. We now present results in the cases where the conjecture holds.

Theorem 6.2 (Blomer-Harcos). When $F$ is a number field, the ternary representation conjecture (Conjecture 6.1) holds for $T=\{v: v \mid \infty\}$ the set of archimedean places with an ineffective constant $c_{Q}$.

Proof. This is almost the statement given by Blomer-Harcos [3, Corollary 2], but where it is assumed that $Q$ is positive definite: we recover the result for $Q$ definite by multiplying $Q$ by two different prime elements with appropriate signs.

Remark 6.3. Using Theorem 6.2, one can show that Conjecture 6.1 holds for all (finite sets) $T$, but we do not need this result in what follows.

In the case where $F$ is a function field, we know of the following partial result.

Theorem 6.4 (Altuğ-Tsimerman, 1, Corollary 1.1]). The ternary representation conjecture holds with an effective constant $c_{Q}$ when $F=\mathbb{F}_{p}(t)$ and $p \equiv 1(\bmod 4)$ and $T=\{\infty\}$.

Discriminants. Define the discriminant quadratic form on $\mathcal{O}$ by

$$
\begin{aligned}
\operatorname{disc}: \mathcal{O} & \rightarrow R \\
\alpha & \mapsto \operatorname{trd}(\alpha)^{2}-4 \operatorname{nrd}(\alpha) .
\end{aligned}
$$

We define similarly $\operatorname{disc}_{\mathfrak{p}}: \mathcal{O}_{(\mathfrak{p})} \rightarrow R_{(\mathfrak{p})}$ for each prime $\mathfrak{p}$.

Lemma 6.6. Let $\mathfrak{p} \subseteq R$ be prime with $S(\mathfrak{p})=R_{(\mathfrak{p})}\left[\alpha_{\mathfrak{p}}\right]$ an integrally closed quadratic order. Let $f \in \mathbb{Z}_{\geq 0}$ be such that $\mathfrak{p}^{2 f} \mid \operatorname{disc}_{\mathfrak{p}}\left(\alpha_{\mathfrak{p}}\right)$. Then there exists a submodule $M \subseteq \mathcal{O}$ such that

(i) $\operatorname{disc}(\beta) \in \mathfrak{p}^{2 f}$ for all $\beta \in M$;

(ii) $M_{(\mathfrak{q})}=\mathcal{O}_{(\mathfrak{q})}$ for $\mathfrak{q} \neq \mathfrak{p}$; and

(iii) $S(\mathfrak{p}) \subseteq M_{(\mathfrak{p})}$.

Proof. First, we have that $\mathcal{O}_{(\mathfrak{p})}$ contains $S(\mathfrak{p})$, which is necessarily integrally closed. Then, $\mathcal{O}_{(\mathfrak{p})} \simeq S(\mathfrak{p})+S(\mathfrak{p}) \gamma$ is an $S(\mathfrak{p})$-module. Moreover, $\mathfrak{p} R_{(\mathfrak{p})}=\pi R_{(\mathfrak{p})}$ is principal. Define $M(\mathfrak{p}):=S(\mathfrak{p})+S(\mathfrak{p}) \pi^{f} \gamma$. For any $\beta \in M(\mathfrak{p})$, we have $\operatorname{disc}(\beta) \in \mathfrak{p}^{2 f} R_{(\mathfrak{p})}$. Then, we define $M:=M(\mathfrak{p}) \cap \mathcal{O} \subseteq \mathcal{O}$. Since $\left(\pi^{f} R_{(\mathfrak{p})} \cap R\right)_{(\mathfrak{q})}=R_{(\mathfrak{q})}$ for all $\mathfrak{q} \neq \mathfrak{p}$, we have $M_{(\mathfrak{q})}=\mathcal{O}_{(\mathfrak{q})}$, and $(M(\mathfrak{p}))_{(\mathfrak{p})}=M_{(\mathfrak{p})}$. Also, $S(\mathfrak{p}) \subseteq M(\mathfrak{p})$, so in particular, we have $S(\mathfrak{p}) \subseteq M_{(\mathfrak{p})}$. 
Lemma 6.7. Suppose $\mathcal{O}_{(\mathfrak{p})}$ is basic for all primes $\mathfrak{p}$. Then there exists an $R$-lattice $M \subseteq \mathcal{O}$, a totally negative $a \in R$, and for every prime $\mathfrak{p}$ elements $\alpha_{\mathfrak{p}} \in M_{(\mathfrak{p})}$ such that $R_{(\mathfrak{p})}\left[\alpha_{\mathfrak{p}}\right]$ is integrally closed and the following conditions hold:

(i) $\left.a^{-1} \operatorname{disc}\right|_{M}: M \rightarrow R$ is a positive definite quadratic form;

(ii) $\left(a^{-1} \operatorname{disc}_{\mathfrak{p}}\right)\left(\alpha_{\mathfrak{p}}\right) \in R_{(\mathfrak{p})}$ is squarefree for every prime $\mathfrak{p}$; and

(iii) $\operatorname{disc}\left(\alpha_{\mathfrak{p}}\right) \in R_{(\mathfrak{p})}^{\times}$for all but finitely many $\mathfrak{p}$.

Proof. For $\mathfrak{p} \mid 2 R$, let $\alpha_{\mathfrak{p}} \in \mathcal{O}_{\mathfrak{p}}$ be such that $v_{\mathfrak{p}}\left(\alpha_{\mathfrak{p}}\right)$ is minimal and let $f_{\mathfrak{p}}$ be the largest integer such that $\mathfrak{p}^{2 f_{\mathfrak{p}}} \mid \operatorname{disc}_{\mathfrak{p}}\left(\alpha_{\mathfrak{p}}\right)$. Similarly, for $\mathfrak{p} \mid 2 R$, let $M^{(\mathfrak{p})} \subseteq \mathcal{O}$ be as in Lemma 6.6 with $\operatorname{disc}(\beta) \in \mathfrak{p}^{2 f_{\mathfrak{p}}} R_{(\mathfrak{p})}$ for all $\beta \in M^{(\mathfrak{p})}$. Define

$$
\mathfrak{b}:=\prod_{\mathfrak{p} \mid 2 R} \mathfrak{p}^{2 f_{\mathfrak{p}}} .
$$

By the Chebotarev density theorem applied to the narrow class field, there exists a prime $\mathfrak{q} \nmid 2 \operatorname{discrd}(\mathcal{O})$ such that $\mathfrak{b q}=a R$ is principal and $a$ is totally negative. Since $\mathfrak{q} \nmid \operatorname{discrd}(\mathcal{O})$, we have $\mathcal{O}_{(\mathfrak{q})} \simeq \mathrm{M}_{2}\left(R_{(\mathfrak{q})}\right)$, so there exists $\alpha_{\mathfrak{q}} \in \mathcal{O}_{(\mathfrak{q})}$ with $v_{\mathfrak{q}}\left(\operatorname{disc}\left(\alpha_{\mathfrak{q}}\right)\right)=1$. Let $\varrho$ be a uniformizer for $R_{(\mathfrak{q})}$, define $M(\mathfrak{q}) \subseteq \mathcal{O}_{(\mathfrak{q})}$ to be the $R_{(\mathfrak{q})}$-suborder with basis

$$
\left(\begin{array}{ll}
1 & 0 \\
0 & 1
\end{array}\right),\left(\begin{array}{cc}
\varrho & 0 \\
0 & -\varrho
\end{array}\right),\left(\begin{array}{cc}
0 & 1 \\
\varrho & 0
\end{array}\right),\left(\begin{array}{cc}
0 & -1 \\
\varrho & 0
\end{array}\right)
$$

all of whose discriminants are divisible by $\varrho$. Define $M^{(\mathfrak{q})}:=M(\mathfrak{q}) \cap \mathcal{O}$. Then $\operatorname{disc}\left(M^{(\mathfrak{q})}\right) \subseteq \mathfrak{q}$. We also have that $\left(M^{(\mathfrak{q})}\right)_{\mathfrak{p}} \simeq \mathcal{O}_{(\mathfrak{p})}$ for all $\mathfrak{p} \neq \mathfrak{q}$ since $\varrho \mathcal{O} \subseteq M^{(\mathfrak{q})}$

For the remaining primes $\mathfrak{p} \nmid 2 a R$, let $\alpha_{\mathfrak{p}} \in \mathcal{O}_{\mathfrak{p}}$ be such that $v_{\mathfrak{p}}\left(\alpha_{\mathfrak{p}}\right)$ is minimal and let $M^{(\mathfrak{p})}:=\mathcal{O}$. Define

$$
M:=\bigcap_{\mathfrak{p}} M^{(\mathfrak{p})} .
$$

By construction we have $\alpha_{\mathfrak{p}} \in M_{(\mathfrak{p})}$ for all $\mathfrak{p}$. Checking locally we have $a \mid \operatorname{disc}(\beta)$ for all $\beta \in M$. We also have that $M_{(\mathfrak{p})}=M^{(\mathfrak{p})}$ for all $\mathfrak{p} \mid a R$ and $M_{(\mathfrak{p})}=\mathcal{O}_{(\mathfrak{p})}$ for all $\mathfrak{p} \nmid a R$. Now, $\left.a^{-1} \operatorname{disc}\right|_{M}: M \rightarrow R$ is positive definite (because disc was negative definite and $a$ was totally negative), so (i) holds.

To conclude, we check (ii) and (iii). Let $e_{\mathfrak{p}}=v_{\mathfrak{p}}\left(a^{-1} \operatorname{disc}_{\mathfrak{p}}\left(\alpha_{\mathfrak{p}}\right)\right)$ for a prime $\mathfrak{p}$. If $\mathfrak{p} \mid 2 R$, then $\mathfrak{p} \mid \mathfrak{b}$ so $e_{\mathfrak{p}} \leq 1$ by construction (we removed the square part). If $\mathfrak{p}=\mathfrak{q}$, by construction $e_{\mathfrak{q}}=0$. Otherwise, since $\mathcal{O}_{\mathfrak{p}}$ is basic and $\mathfrak{p} \nmid 2 a R$, we have $e_{\mathfrak{p}} \leq 1$. In particular, $e_{p}=0$ for all but finitely many $\mathfrak{p}$, so (iii) holds.

We give a final lemma before proving the theorem.

Lemma 6.8. Suppose B is T-definite and that Conjecture 6.1 holds over $R$. Let $\mathcal{O} \subseteq B$ an $R$-order such that $\mathcal{O}_{(\mathfrak{p})}$ is basic for every prime $\mathfrak{p}$ of $R$. Then $\mathcal{O}$ contains infinitely many nonisomorphic integrally closed free quadratic $R$-orders.

Proof. By Lemma 6.7 we obtain the following: an $R$-lattice $M \subseteq \mathcal{O}$, a totally negative $a \in R$, and for every prime $\mathfrak{p}$ elements $\alpha_{\mathfrak{p}} \in M_{(\mathfrak{p})}$ such that $R_{(\mathfrak{p})}\left[\alpha_{\mathfrak{p}}\right]$ is integrally closed and the conditions (i)-(iii) hold.

For each $\mathfrak{p}$, let $d_{\mathfrak{p}}:=\operatorname{disc}\left(\alpha_{\mathfrak{p}}\right)$ and $e_{\mathfrak{p}}:=v_{\mathfrak{p}}\left(d_{\mathfrak{p}}\right)$. Define $\mathfrak{d}:=\prod_{\mathfrak{p}} \mathfrak{p}^{e_{\mathfrak{p}}}$. Note that if $\mathfrak{p}^{e} \mid a R$ then $\mathfrak{p}^{e} \mid d_{\mathfrak{p}}$, so $\mathfrak{p}^{e} \mid \mathfrak{d}$. Therefore, $a R \mid \mathfrak{d}$.

By the Chebotarev density theorem applied to the narrow Hilbert class field, there exists a prime $\mathfrak{q} \nmid 2 \mathfrak{d}$ such that $\mathfrak{d} \mathfrak{q}=m R$ is principal and $m$ is totally negative. In particular, $a \mid m$. Define $t_{\mathfrak{p}}$ as in (5.2) and $\mathfrak{n}$ as in (5.3). Applying the Chebotarev density theorem again, this time to the ray class field with conductor $\mathfrak{n}$, there exist 
totally positive prime elements $\pi \nmid m$ with arbitrarily large absolute norm such that $\pi \equiv m^{-1} d_{\mathfrak{p}}\left(\bmod \mathfrak{p}^{t_{\mathfrak{p}}}\right)$ for all $\mathfrak{p} \mid 2 \mathfrak{d}$. Let $d:=\pi m$. Then $a^{-1} d$ is totally positive and squarefree by construction, and there are infinitely many such choices.

Let $d$ be such a discriminant. We claim that $d$ is locally represented by $\left.\operatorname{disc}\right|_{M}$. Indeed, we have $\alpha_{\mathfrak{p}} \in M_{(\mathfrak{p})}$ for all $\mathfrak{p}$ by construction. For $\mathfrak{p} \neq \mathfrak{q}, \pi R$, we have

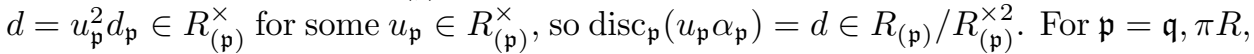
we have $\mathfrak{p} \nmid 2 \operatorname{discrd}(\mathcal{O})$, so $M_{(\mathfrak{p})}=\mathcal{O}_{(\mathfrak{p})} \simeq \mathrm{M}_{2}\left(R_{(\mathfrak{p})}\right)$, so $\operatorname{disc}_{\mathfrak{p}}: \mathcal{O}_{(\mathfrak{p})} \rightarrow R_{(\mathfrak{p})}$ is surjective; in particular $\operatorname{disc}_{\mathfrak{p}}$ represents $d$.

Therefore $a^{-1} d$ is locally represented by $a^{-1}$ disc $\left.\right|_{M}$. Therefore, if the conclusion of Conjecture 6.1 holds, taking $d$ to be of sufficiently large norm, there is an element $\alpha \in M \subseteq \mathcal{O}$ with $a^{-1} \operatorname{disc}(\alpha)=a^{-1} d$, so $\operatorname{disc}(\alpha)=d$.

Finally, let $S_{d}:=R[\alpha] \subseteq \mathcal{O}$. For $\mathfrak{p} \mid \operatorname{discrd}(\mathcal{O})$, we have that $\left(S_{d}\right)_{(\mathfrak{p})}=R_{(\mathfrak{p})}[\alpha]=$ $R_{(\mathfrak{p})}\left[\alpha_{\mathfrak{p}}\right]$ is maximal in its field of fractions by construction. For $\mathfrak{p} \nmid \operatorname{discrd}(\mathcal{O})$, we have $v_{\mathfrak{p}}(d) \leq 1$, so again $\left(S_{d}\right)_{(\mathfrak{p})}=R_{(\mathfrak{p})}[\alpha]$ is maximal in its field of fractions. Therefore, $S_{d}$ is maximal in its field of fractions and so $\mathcal{O}$ is basic.

We now prove Theorem 1.2 in the definite case for $R$ the ring of integers of a number field.

Proof of Theorem 1.2, $B$ definite, $R$ the ring of integers of a number field. For part (a), if $\mathcal{O}_{(\mathfrak{p})}$ is basic for every prime $\mathfrak{p}$ of $R$, then $\mathcal{O}$ contains an integrally closed quadratic $R$-order by Lemma 6.8 using Theorem 6.2. The converse is exactly as in the proof of Theorem 1.2 in the indefinite case, as given in Section 5 .

Being Bass is a local property, and local orders are basic if and only if they are Bass by Theorem 3.12 so (b) follows from (a).

Corollary 6.9. Suppose that $B$ is $T$-definite and let $\mathcal{O} \subseteq B$ be an $R$-order. If $\mathcal{O}$ is basic, then $\mathcal{O}$ contains infinitely many nonisomorphic integrally closed quadratic $R$-orders.

Proof. Combine Theorem 1.2 in the definite case with Lemma 6.8 .

\section{LOCALIZATIONS}

We conclude the proof of Theorem 1.2 by deducing the basic property of an order over a Dedekind domain from that of its localizations. Throughout, let $R$ be a Dedekind domain with $F=\operatorname{Frac} R$ and let $\mathcal{O}$ be a quaternion $R$-order.

Lemma 7.1. Let $R^{\prime} \subseteq R$ be Dedekind domains such that $F:=\operatorname{Frac}(R)=\operatorname{Frac}\left(R^{\prime}\right)$ is a global field. Let $\mathcal{O}$ be an $R$-order. Then there is an $R^{\prime}$-order $\mathcal{O}^{\prime} \subseteq \mathcal{O}$ such that $\mathcal{O}=\mathcal{O}^{\prime} R$ and

- $\mathcal{O}_{(\mathfrak{p})}^{\prime}=\mathcal{O}_{(\mathfrak{p})}$ for every prime $\mathfrak{p}$ of $R^{\prime}$ with $\mathfrak{p} R \neq R$,

- $\mathcal{O}_{(\mathfrak{p})}^{\prime}$ is a maximal order for every prime $\mathfrak{p}$ of $R^{\prime}$ with $\mathfrak{p} R=R$.

In particular, if $\mathcal{O}$ Bass, then $\mathcal{O}^{\prime}$ is Bass.

Proof. Since $R$ and $R^{\prime}$ are necessarily overrings of a global ring, their class groups are finite. It follows that there exists a multiplicative set $S \subseteq R^{\prime}$ such that $R=$ $S^{-1} R^{\prime}$ [9, Theorem 5.5]. Let $\alpha_{1}, \ldots, \alpha_{m}$ be generators for the $R$-module $\mathcal{O}$. There exists (a common denominator) $d \in S$ such that

$$
d \alpha_{i} \alpha_{j} \in R^{\prime} \alpha_{1}+\cdots+R^{\prime} \alpha_{m} \quad \text { for all } i, j=1, \ldots, m \text {. }
$$


This implies $\left(d \alpha_{i}\right)\left(d \alpha_{j}\right) \in R^{\prime} d \alpha_{1}+\cdots+R^{\prime} d \alpha_{m}$. Thus $d \alpha_{1}, \ldots, d \alpha_{m}$ generate an $R^{\prime}$-order $\mathcal{O}^{\prime \prime} \subseteq \mathcal{O}$ with $R \mathcal{O}^{\prime \prime}=\mathcal{O}$. In particular, $\mathcal{O}_{(\mathfrak{p})}^{\prime \prime}=\mathcal{O}_{(\mathfrak{p})}$ for every prime $\mathfrak{p}$ of $R^{\prime}$ with $\mathfrak{p} \cap S=\emptyset$. Let $\mathcal{P}$ be the set of prime ideals $\mathfrak{p}$ of $R^{\prime}$ with $\mathfrak{p} \cap S \neq \emptyset$ for which $\mathcal{O}_{(\mathfrak{p})}^{\prime \prime}$ is not maximal. Since any $\mathfrak{p} \in \mathcal{P}$ has $\mathfrak{p} \mid \operatorname{discrd}\left(\mathcal{O}^{\prime \prime}\right)$, the set $\mathcal{P}$ is finite. By the local-global dictionary for lattices, there exists an $R^{\prime}$-order $\mathcal{O}^{\prime}$ with $\mathcal{O}^{\prime \prime} \subseteq \mathcal{O}^{\prime}$ such that $\mathcal{O}_{\left(\mathfrak{p}^{\prime}\right)}=\mathcal{O}_{\left(\mathfrak{p}^{\prime \prime}\right)}$ for all $\mathfrak{p} \notin \mathcal{P}$ and $\mathcal{O}_{(\mathfrak{p})}^{\prime}$ is maximal for $\mathfrak{p} \in \mathcal{P}$. Since $\mathcal{O}_{\left(\mathfrak{p}^{\prime}\right)}=\mathcal{O}_{\left(\mathfrak{p}^{\prime \prime}\right)} \subseteq \mathcal{O}_{(\mathfrak{p})}$ for all primes $\mathfrak{p}$ of $R$ with $\mathfrak{p} \cap S=\emptyset$, we still have $\mathcal{O}^{\prime} \subseteq \mathcal{O}$.

Since being Bass is a local property, and at all $\mathfrak{p}$ of $R$ we have either $\mathcal{O}_{(\mathfrak{p})}^{\prime}$ maximal or equal to $\mathcal{O}_{(\mathfrak{p})}$, the order $\mathcal{O}^{\prime}$ is Bass.

Lemma 7.3. Suppose $F=\operatorname{Frac}(R)$ is a global field, and let $T$ be the (nonempty) set of places of $F$ such that $R=R_{(T)}$. Suppose $\# T=\infty$. If $\mathcal{O}$ is Bass, there exist infinitely many nonisomorphic maximal quadratic $R$-orders $S$ that embed into $\mathcal{O}$.

Proof. Since $T$ is infinite, there exists a place $v \in T$ such that $B_{v}$ is unramified. Let $T^{\prime}$ be a finite set of places containing $v$ and all archimedean places of $F$. By

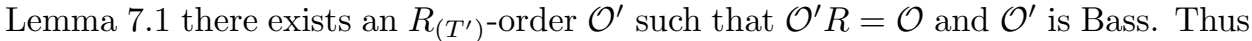
$\mathcal{O}^{\prime}$ is locally Bass and hence locally basic by Theorem 1.1 since $\mathcal{O}^{\prime}$ is $T$-indefinite, Lemma 5.5implies that there are infinitely many nonisomorphic maximal quadratic $R^{\prime}$-orders $S^{\prime} \hookrightarrow \mathcal{O}^{\prime}$, with each $S:=R S^{\prime}$ a maximal, quadratic $R$-order that embeds in $\mathcal{O}$. Thus there are infinitely many nonisomorphic such orders $S$.

Lemma 7.4. Let $R^{\prime} \subseteq R$ be Dedekind domains with $\operatorname{Frac}\left(R^{\prime}\right)=\operatorname{Frac}(R)$ a global field. Suppose that every $R^{\prime}$-order that is Bass is basic. Then every $R$-order that is Bass is basic.

Proof. As in Lemma 7.3 .

Theorem 7.5. Suppose that $F=\operatorname{Frac}(R)$ is a global field. Let $T$ be the nonempty (possibly infinite) set of places such that $R=R_{(T)}$. Let $\mathcal{O}$ be an $R$-order. Suppose that one of the following conditions holds:

(i) $F$ is a number field;

(ii) $\# T<\infty$ and $B$ is $T$-indefinite; or

(iii) $\# T=\infty$.

Then the following statements hold.

(a) $\mathcal{O}$ is basic if and only if the localization $\mathcal{O}_{(\mathfrak{p})}$ is basic for all primes $\mathfrak{p}$ of $R$.

(b) $\mathcal{O}$ is Bass if and only if $\mathcal{O}$ is basic.

Proof. Being Bass is a local property, and local orders are basic if and only if they are Bass by Theorem 3.12. Thus it suffices to show (b). Basic orders are Bass by Proposition 3.4 and we are left to show that an $R$-order $\mathcal{O}$ that is Bass is basic.

Suppose first that $\# T<\infty$. If $B$ is $T$-indefinite, the claim follows from Theorem 1.2 in the indefinite case, as proved in Section 5. Suppose that $F$ is a number field and $B$ is $T$-definite. Let $T^{\prime}$ be the set of all archimedean places of $F$. Then $R_{\left(T^{\prime}\right)}$ is the ring of integers of $F$, and the claim holds by the proof of Theorem 1.2 for the definite case in Section 6 together with Theorem 6.2. Lemma 7.4 shows that the result also holds for $R_{(T)}$.

Finally, if $\# T=\infty$, apply Lemma 7.3 .

Proof of Theorem 1.2. Restrict Theorem 7.5 to the case $F$ is a number field. 
Corollary 7.6. If one of the conditions in Theorem 7.5)(i)-(iii) holds, and $\mathcal{O}$ is basic, then $\mathcal{O}$ contains infinitely many nonisomorphic integrally closed free quadratic $R$-orders.

Proof. Corollaries 5.6 and 6.9 for $\# T<\infty$ and Lemma 7.3 for $\# T=\infty$.

\section{ACKNOWLEDGMENTS}

The authors would like to thank Wai Kiu Chan and Tom Shemanske for helpful conversations.

\section{REFERENCES}

[1] Salim Ali Altuğ and Jacob Tsimerman, Metaplectic Ramanujan conjecture over function fields with applications to quadratic forms, Int. Math. Res. Not. IMRN 13 (2014), 34653558, DOI 10.1093/imrn/rnt047. MR3229761

[2] Hyman Bass, On the ubiquity of Gorenstein rings, Math. Z. 82 (1963), 8-28, DOI 10.1007/BF01112819. MR153708

[3] Valentin Blomer and Gergely Harcos, Twisted L-functions over number fields and Hilbert's eleventh problem, Geom. Funct. Anal. 20 (2010), no. 1, 1-52, DOI 10.1007/s00039-010-0063-x. $\operatorname{MR} 2647133$

[4] Juliusz Brzeziński, A characterization of Gorenstein orders in quaternion algebras, Math. Scand. 50 (1982), no. 1, 19-24, DOI 10.7146/math.scand.a-11940. MR664504

[5] J. Brzezinski, On automorphisms of quaternion orders, J. Reine Angew. Math. 403 (1990), 166-186, DOI 10.1515/crll.1990.403.166. MR1030414

[6] James W. Cogdell, On sums of three squares (English, with English and French summaries), J. Théor. Nombres Bordeaux 15 (2003), no. 1, 33-44. Les XXIIèmes Journées Arithmetiques (Lille, 2001). MR2018999

[7] Ju. A. Drozd, V. V. Kiričenko, and A. V. Roŭter, Hereditary and Bass orders (Russian), Izv. Akad. Nauk SSSR Ser. Mat. 31 (1967), 1415-1436. MR0219527

[8] Martin Eichler, Untersuchungen in der Zahlentheorie der rationalen Quaternionenalgebren (German), J. Reine Angew. Math. 174 (1936), 129-159, DOI 10.1515/crll.1936.174.129. MR.1581481

[9] Stefania Gabelli, Generalized Dedekind domains, Multiplicative ideal theory in commutative algebra, Springer, New York, 2006, pp. 189-206, DOI 10.1007/978-0-387-36717-0_12. MR2265809

[10] Benedict H. Gross and Mark W. Lucianovic, On cubic rings and quaternion rings, J. Number Theory 129 (2009), no. 6, 1468-1478, DOI 10.1016/j.jnt.2008.06.003. MR2521487

[11] Hiroaki Hijikata, Arnold K. Pizer, and Thomas R. Shemanske, The basis problem for modular forms on $\Gamma_{0}(N)$, Mem. Amer. Math. Soc. 82 (1989), no. 418, vi+159, DOI 10.1090/memo/0418. MR.960090

[12] H. Hijikata, A. Pizer, and T. Shemanske, Orders in quaternion algebras, J. Reine Angew. Math. 394 (1989), 59-106. MR.977435

[13] Sungtae Jun, On the certain primitive orders, J. Korean Math. Soc. 34 (1997), no. 4, 791-807. MR.1485952

[14] Arnold Pizer, The action of the canonical involution on modular forms of weight 2 on $\Gamma_{0}(M)$, Math. Ann. 226 (1977), no. 2, 99-116, DOI 10.1007/BF01360861. MR437463

[15] Rainer Schulze-Pillot, Representation by integral quadratic forms - a survey, Algebraic and arithmetic theory of quadratic forms, Contemp. Math., vol. 344, Amer. Math. Soc., Providence, RI, 2004, pp. 303-321, DOI 10.1090/conm/344/06226. MR2060206

[16] John Voight, Identifying the matrix ring: algorithms for quaternion algebras and quadratic forms, Quadratic and higher degree forms, Dev. Math., vol. 31, Springer, New York, 2013, pp. 255-298, DOI 10.1007/978-1-4614-7488-3_10. MR3156561

[17] John Voight, Quaternion algebras, Grad. Texts. in Math., vol. 288, Springer-Verlag, New York, 2020. 
Department of Mathematics, Dartmouth College, 6188 Kemeny Hall, Hanover, New HAMPSHIRE 03755

Email address: schari0301@gmail.com

$U R L:$ https://www.math.dartmouth.edu/ schari/

Department of Pure Mathematics, University of Waterloo, Waterloo, Ontario, N2L 3G1 CANADA

Email address: dsmertni@uwaterloo.ca

URL: https://www.math.uwaterloo.ca/ dsmertni/

Department of Mathematics, Dartmouth College, 6188 Kemeny Hall, Hanover, New HAMPSHIRE 03755

Email address: jvoight@gmail.com

URL: http://www.math.dartmouth.edu/ jvoight/ 